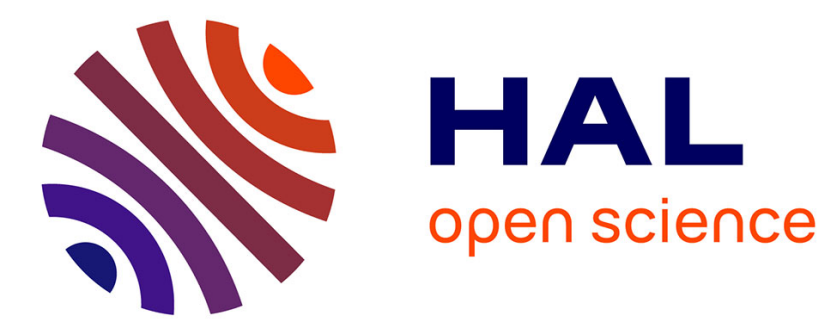

\title{
Spectromètre de paires et anti-Compton
}

\author{
J. Girard, R. Samama, P. Carlos, B.P. Maier, G. Perrin
}

\section{To cite this version:}

J. Girard, R. Samama, P. Carlos, B.P. Maier, G. Perrin. Spectromètre de paires et anti-Compton. Revue de Physique Appliquée, 1969, 4 (2), pp.261-262. 10.1051/rphysap:0196900402026100 . jpa00243251

\section{HAL Id: jpa-00243251 https://hal.science/jpa-00243251}

Submitted on 1 Jan 1969

HAL is a multi-disciplinary open access archive for the deposit and dissemination of scientific research documents, whether they are published or not. The documents may come from teaching and research institutions in France or abroad, or from public or private research centers.
L'archive ouverte pluridisciplinaire HAL, est destinée au dépôt et à la diffusion de documents scientifiques de niveau recherche, publiés ou non, émanant des établissements d'enseignement et de recherche français ou étrangers, des laboratoires publics ou privés. 


\title{
SPECTROMÈTRE DE PAIRES ET ANTI-COMPTON
}

\author{
J. GIRARD, R. SAMAMA, P. GARLOS, B. P. MAIER et G. PERRIN, \\ Service des Mesures Neutroniques Fondamentales, C.E.N., Saclay, France.
}

\begin{abstract}
Résumé. - Nous décrivons un ensemble pouvant fonctionner en spectromètre de paires ou en anti-Compton. Il est constitué d'une diode au $\mathrm{Ge}(\mathrm{Li})$ de $10 \mathrm{~cm}^{3}$ et d'un cristal annulaire de $\mathrm{INa}(\mathrm{Tl})$ divisé en 6 secteurs vus chacun par un photomultiplicateur. Les signaux issus du scintillateur sont utilisés pour le conditionnement de l'information fournie par la diode.
\end{abstract}

Abstract. - A gamma ray spectrometer working either in the anti-Compton or in the pair mode is described. It is composed by a $10 \mathrm{~cm}^{3} \mathrm{Ge}(\mathrm{Li})$ detector surrounded by a large $\mathrm{INa}(\mathrm{Tl})$ annulus which is divided in 6 sectors. The pulses coming from these sectors are combined and used to condition the analysis of the pulses detected by the $\mathrm{Ge}(\mathrm{Li})$ detector.

Nous décrivons un spectromètre fonctionnant en anti-Compton ou paires. Il se compose d'un détecteur $\mathrm{Ge}(\mathrm{Li})$ de $10 \mathrm{~cm}^{3}$ pour une profondeur de drift de $1 \mathrm{~cm}$, présentant une résolution de $5,6 \mathrm{keV}$ à $1,332 \mathrm{MeV}$ et de $11 \mathrm{keV}$ à $7 \mathrm{MeV}$, placé au centre d'un cristal annulaire $\mathrm{INa}(\mathrm{Tl})$ de $10^{\prime \prime} \times 12^{\prime \prime}$ divisé en six secteurs opposés par le sommet et optiquement isolés. Chacun de ces six secteurs est vu par un photomultiplicateur. Les impulsions délivrées par la diode $\mathrm{Ge}(\mathrm{Li})$ sont amplifiées et codées par un codeur analogique digital et transmises à un analyseur multicanal à 4096 canaux. La figure 1 montre le diagramme logique de notre installation.

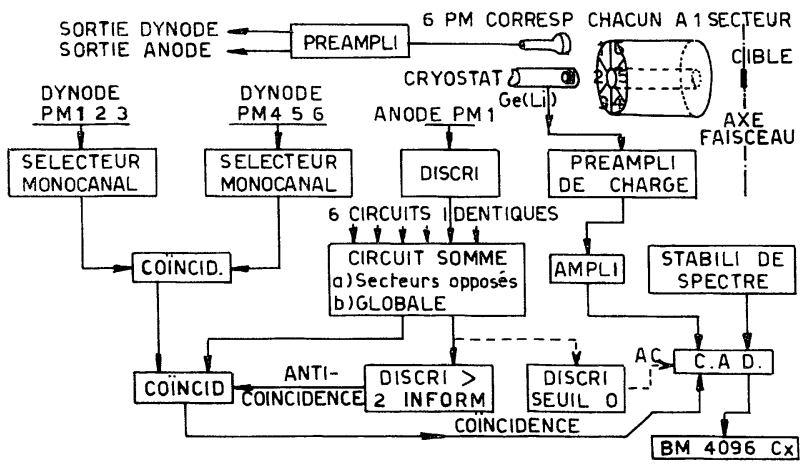

Fig. 1. - Schéma synoptique de l'expérience $\gamma$ de capture.

I. Spectrométrie anti-Compton. - Jusqu'à quelques $\mathrm{MeV}$, l'effet Compton est très important et sa contribution dans les spectres est gênante pour l'analyse des raies situées à ces énergies. Nous ne désirons conserver l'information que si elle correspond à un effet photoélectrique. Dans le cas d'un effet Compton, le rayon $\gamma$ diffusé a une grande probabilité d'être détecté par l'ensemble scintillant; nous rejetons alors l'informaticn.

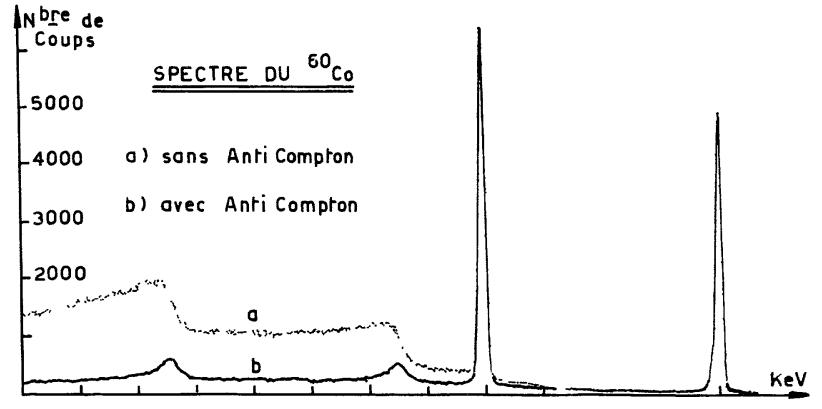

FIG. 2. - Spectre de ${ }^{60} \mathrm{Co}$.

La figure 2 compare les spectres de rayons $\gamma$ d'une source radioactive de ${ }^{60} \mathrm{Co}$ obtenus avec et sans cette méthode. Le fond Compton est ainsi diminué d'un facteur 6 pour les énergies supérieures à $400 \mathrm{keV}$ environ. L'amélioration n'est pas uniforme dans tout le spectre pour des raisons essentiellement géométriques : 1) les rayons $\gamma$ rétrodiffusés ne rencontrant pas le cristal, nous retrouvons à l'emplacement des fronts Compton une raie très dissymétrique sur son côté gauche; 2) de même, les rayons $\gamma$ diffusés vers l'avant ne sont pas vus par l'ensemble scintillant, d'où une amélioration moins nette de la partie du spectre située jusqu'à quelques centaines de keV.

Le rapport entre le taux de comptage au pic de la raie à $1332 \mathrm{keV}$ et le taux de comptage dans le bruit Compton correspondant à cette raie est dans notre cas de l'ordre de 25 , alors qu'il n'est que de 5 en fonctionnement normal.

II. Spectrométrie de paires. - Au-delà de $1,02 \mathrm{MeV}$, les effets photoélectrique, Compton et création de paires sont possibles. Ce dernier devient prépondérant dès $2 \mathrm{MeV}$ et s'accompagne de l'émission de quanta 
de $511 \mathrm{keV}$ en opposition provenant de l'annihilation du positon. La diode donne alors, pour des photons monochromatiques, un spectre comportant, sur un fond Compton et de « bremsstrahlung », trois pics : le plus haut en énergie comprend les effets photoélectriques et de création de paires avec arrêt des deux quanta d'annihilation dans la diode. Les deux autres correspondent à l'échappement de l'un ou des deux photons de $511 \mathrm{keV}$.

Il est souhaitable, afin de simplifier l'étude des spectres, de ne conserver que les raies de double échappement. Pour cela, l'information détectée par la diode n'est conservée que si les conditions suivantes sont réalisées : 1) chaque demi-couronne formée par trois secteurs a détecté un quantum de $511 \mathrm{keV}$ sélectionné par un discriminateur à bande; 2) deux secteurs opposés et deux seulement ont détecté une radiation; ceci dans le but d'éliminer les effets de diffusions multiples et de «bremsstrahlung ». La perte d'efficacité de détection, dans les pics de double échappement, résultat de ce conditionnement, est de 6 . Elle correspond à l'efficacité de détection au pic photoélectrique de deux rayons $\gamma$ de $511 \mathrm{keV}$ en opposition par l'ensemble scintillant.
Cet appareillage nous a permis d'étudier la réaction ${ }^{62} \mathrm{Ni}(\mathrm{n}, \gamma)^{63} \mathrm{Ni}$ à basse énergie en anti-Compton et à haute énergie en paires. Nous avons pu établir une proposition de schéma en utilisant des règles de somme, mais il était utile de confirmer les cascades par des mesures en coïncidence. Ces mesures ont été réalisées avec un détecteur $\mathrm{Ge}(\mathrm{Li})$ de $10 \mathrm{~cm}^{3}$ sans conditions particulières et un photomultiplicateur équipé d'un scintillateur $\mathrm{INa}(\mathrm{Tl})$ de $3^{\prime \prime} \times 4^{\prime \prime}$ placé au centre de la couronne citée précédemment. Ce dernier ensemble fonctionnait en anti-Compton. Gette expérience nous a permis de confirmer certaines cascades suggérées par les règles de sommes, mais par contre, pour plusieurs raisons, nous n'avons pu en mettre de nouvelles en évidence : 1) le photomultiplicateur ne possédait pas une résolution suffisante : $7,5 \%$ sur ${ }^{60} \mathrm{Co} ; 2$ ) le spectre obtenu avec la diode comportait trop d'effets parasites : Compton, pics de simple et double échappement.

Nous pensons reprendre ces expériences multiparamétriques de coïncidence entre deux diodes $\mathrm{Ge}(\mathrm{Li})$. L'une, associée à un cristal INa de fort diamètre situé immédiatement derrière, fonctionnera en anti-Compton. L'autre de plusieurs dizaines de $\mathrm{cm}^{3}$, placée au centre de la couronne, fonctionnera en paires. 